\title{
Dither-Less Extremum Seeking for Hydrogen Minimization in PEM Fuel Cells
}

\author{
Fernando Castaños, Member IEEE and Cristian Kunusch, Member IEEE
}

\begin{abstract}
This work presents a nonsmooth adaptive extremum seeker that minimizes the hydrogen consumption in a fuel cell system. The extremum seeker operates by estimating the gradient of the objective function but, unlike other seekers, it does not require a dither signal to produce such estimate. The absence of a dither signal simplifies the choice of parameter values for the seeker and, more importantly, it allows it to converge to the optimal value exactly, not only to a small neighborhood. The proper functioning of the proposed scheme is proved using nonsmooth Lyapunov analysis. The strategy is tested on the input-output map of a real PEM fuel cell.
\end{abstract}

Index Terms-Extremum Seeking; Fuel Cells; Nonsmooth Lyapunov Analysis.

\section{INTRODUCTION}

D ESPITE the notorious advantages of polymer electrolyte fuel cells (PEMFC) and the widespread availability of hydrogen as a fuel, several technological challenges related to the PEMFC efficiency, lifetime and economical costs are still open as major limitations for their standard implementation in everyday solutions. This fact, together with the recent advances in materials development and component enhancements, make advanced control techniques appear as the necessary complementary strategies in order to reduce costs, improve performance and optimize efficiency, therefore increasing the lifetime of PEMFC-based systems. Reliable control systems may ensure system stability and performance, as well as robustness against uncertainties and exogenous perturbations, all properties of capital importance for PEMFC success and future industrial development. Several research works have addressed the oxygen stoichiometry through air flow control, avoiding performance deterioration together with eventual irreversible damages in the polymeric membranes due to oxygen starvation. These works present the way to achieve the aforementioned control objective by using different techniques: Model Predictive Control (MPC) [1], Sliding-Mode Control [2], Full-state Feedback with Integral Control [3], Linear Parameter Varying (LPV) control [4], adaptive control

Copyright (c) 2015 IEEE. Personal use of this material is permitted. However, permission to use this material for any other purposes must be obtained from the IEEE by sending a request to pubs-permissions@ieee.org.

Fernando Castaños is with Centro de Investigación y de Estudios Avanzados del Instituto Politécnico Nacional (Cinvestav-IPN), Mexico D.F., Mexico. castanos@ieee. org.

C. Kunusch is with Universitat Politècnica de Catalunya, Institut de Robòtica i Informàtica Industrial (CSIC-UPC), Llorens i Artigas, 4-6, 08028 Barcelona, Spain. ckunusch@iri.upc.edu

The research of C. Kunusch has been supported by the Seventh Framework Programme of the European Community through the Marie Curie actions (GA: PCIG09-GA-2011-293876), the Puma-Mind project (GA: FCH-JU-2011-1303419), the CICYT project DPI2011-25649 (MINECO-Spain), the CSIC MACPERCON project (201250E027) and the CSIC JAE-DOC Research Programme.
[5], among others. Nevertheless, there still lacks rigorous results that solve on-line reference computation for optimal energy efficiency in variable operating conditions. Tracking a varying maximum or minimum (extremum) of a performance (output, cost) function is called extremum seeking control, which has usually two layers of control. The first is an algorithm able to control (stabilize) the system and drive the performance output to its reference. Then, if possible, it is necessary to seek an extremum of the performance output.

In some applications, the reference-to-output map has an extremum (w.l.o.g. we assume that it is a minimum) and the objective is to select the set point to keep the output at the extremum value. The uncertainty in the reference-to-output map makes it necessary to use some sort of adaptation to find the set point which optimizes the output [6].

A thorough historical account of extremum seeking can be found in [7]. The first extremum seeker is traced back to 1922. During the 1950's and 1960's, extremum control is intensively developed by the adaptive control community. From 1970 to 2000 , extremum control is no longer part of the mainstream, but continues to make steady progress. The first rigorous proof of the local stability of an extremum seeking control scheme appears in 2000 [6]. The classical Blackman scheme (where a low-pass filter, a high-pass filter and a slow perturbation signal are employed to extract information abut the gradient of the cost function) is analyzed using averaging analysis and singular perturbation (time-scale separation) techniques. This result appears to have sparked a renewed interest in extremum seeking control.

Extremum seekers usually include some form of perturbation or dither signal. Current extremum seekers can thus be classified according to the nature of such signal, which can either be deterministic (usually periodic) or stochastic. The excitation signal does not necessarily have to be external, it can be a naturally occurring signal (such as noise) with appropriate spectral content. In any case, dither signals have been considered for a long time as essential components of extremum seeking controllers (see [7] and references therein for more details), while considerable efforts have been devoted in the last decade to analyze the degrading effect that dither signals have on the accuracy of the closed-loop system [8], [9].

Most of the results available on extremum control consider a plant as a static map. A few references approach problems where the plant is a cascade of a nonlinear static map and a linear dynamic system (the so-called Hammerstein and Wiener models) (see Wittenmark and Urquhart, 1995 and references therein). In this paper we approach the problem where the nonlinearity with an extremum is the reference-to-output map 
of a general nonlinear (non-affine in the control) system. More precisely, an equilibrium state and its corresponding output are associated to each reference, and the equilibria are stabilizable by a local feedback controller [6].

Applications of extremum control are among the following: combustion processes (for IC engines, steam generating plants, and gas furnaces), grinding processes, solar cell and radio telescope antenna adjustment to maximize the received signal, and blade adjustment in water turbines and wind mills to maximize the generated power. Many more applications are reported in [7]. The authors only found a few references about extremum seeking control applied to fuel cell optimization in the literature. In the work [10], promising initial results considering a second-order sliding-mode algorithm are provided for a PEM fuel cell system simulation model. Nevertheless, no rigorous proof of convergence is given for the seeker and the persistence in the excitation problem is not considered. Therefore it is still difficult to analyze the algorithm's robustness. In [11], an extremum seeking algorithm is proposed based on [6]. In that reference, simulation results are provided for a PEM fuel cell, but there are no hints about the algorithm tuning (specially the dither signal conditions) and its robustness is not analyzed.

\section{SySTEM DESCRIPTION}

The system under study is comprised by a central PEMFC stack and additional/auxiliary units. Fig. 1 shows the scheme of the system and the interaction between its different subsystems (FC stack, reactant supply system and humidity management unit). A brief description of some components, variables and processes is presented as follows. In the system, the main control input corresponds to the compressor voltage, denoted $V_{c p}$. One important system output corresponds in turn to the inlet oxygen mass flow in the PEMFC cathode, namely $W_{c p}$. While the system is affected by an external measurable disturbance $P_{n e t}$, which corresponds to the system net power delivered to the load.

The main subsystems depicted in Fig. 1 are:

- A $12 \mathrm{~V}$ DC air compressor with an oil-free diaphragm vacuum pump, whose input voltage $V_{c p}$ is the control variable (as established beforehand).

- Hydrogen and oxygen membrane exchange humidifiers and line heaters, which are used to maintain proper humidity and temperature conditions inside the cell stack ${ }^{1}$.

- A ZBT ${ }^{\circledR} 8$ fuel-cell stack with Nafion $115^{\circledR}$ membrane electrode assemblies with $50 \mathrm{~cm}^{2}$ of active area and $150 \mathrm{~W}$ power.

Different sensors are incorporated into the system, such as an air-mass flow-meter (range 0-15 slpm) at the end of the compressor to measure its flow $\left(W_{c p}\right)$, a current clamp (range 0-3 A) and a voltage meter (range 0-15 V) to measure the motor stator current $\left(I_{c p}\right)$ and voltage $\left(V_{c p}\right)$, respectively. Besides, temperature sensors are arranged in order to register

\footnotetext{
${ }^{1}$ Decentralized controllers ensure the adequate operation values for these devices, therefore the temperature and humidity control design is out of the scope of this paper.
}

the different operating conditions. In the sequel, the following working conditions are assumed in the test bench:

- The compressor behaves as a parasitic load to the stack.

- A mass flow control device ensures a constant hydrogen stoichiometry close to 1.5 .

- An auxiliary control system efficiently regulates temperatures at five points of the plant: cathode and anode humidifiers $\left(T_{h u m, c a}\right.$ and $\left.T_{h u m, a n}\right)$, cathode and anode line heaters $\left(T_{l h, c a}\right.$ and $\left.T_{l h, a n}\right)$ and stack $\left(T_{s t}\right)$.

- The air mass flow meter has a time constant of $20 \mathrm{~ms}$, so no significant delays are involved in the control loops.

- A humidity control loop regulates the water injection of the humidifiers (anode and cathode) up to a relative level of $100 \%$ at $60^{\circ} \mathrm{C}$.

- The fuel cell model is finite-dimensional, so the gases are considered uniformly distributed in the cell.

- The electrochemical properties are evaluated at the average stack temperature $\left(70^{\circ} \mathrm{C}\right)$, so temperature variations across the stack are neglected.

- The water entering the cathode and the anode are in vapour phase.

- The effects of liquid water creation are negligible at the gas flow model level.

The full description of this system, as well as a fullyvalidated nonlinear dynamic model specially developed for control purposes, are presented and deeply discussed in [12].

In order to maximize the efficiency of the PEMFC system, the regulation of the oxygen mass inflow towards the stack needs to be tackled. As a consequence of a correct regulation of this variable, the oxygen excess ratio [13] is driven to the optimal value and the load demand is satisfied with minimum fuel consumption [14], [15], [16]. Additionally, oxygen starvation and irreversible membrane damages are averted. In this context, the fuel cell oxygen mass flow is described as follows:

$$
W_{\mathrm{O}_{2}, c a}=\frac{\chi_{\mathrm{O}_{2}} W_{c p}}{1+\omega_{a m b}},
$$

where $\omega_{a m b}$ is the ambient air humidity ratio and $\chi_{O_{2}}$ is the molar fraction of oxygen in the air $\left(\chi_{\mathrm{O}_{2}}=0.21\right)$. As presented in the validated model [12], for set temperature and humidity conditions, the operating conditions of the system are determined by the compressor voltage $\left(V_{c p}\right)$ and the net power extracted from the system $\left(P_{n e t}\right)$.

It follows from the chemistry of the fuel cell that higher air/oxygen flow rates translate into higher stack voltage (hence lower load current for the same stack power). On the other hand, higher air/oxygen flow rates imply higher compressor electrical consumption, so a right compromise has to be found in order to minimize this parasitic behaviour. This means that there is a certain optimal value of $W_{c p}$ that makes the stack have the maximum voltage. If the stack is in its maximum voltage, for constant power, the stack current is in its minimum. Note that the minimization of the stack current for a fixed net power is equivalent to the net power maximization for a fixed stack current (point of maximum efficiency).

The work of this paper is focused on the oxygen mass flow $W_{\mathrm{O}_{2}, \text { ca }}$ reference computation under changes in the net 


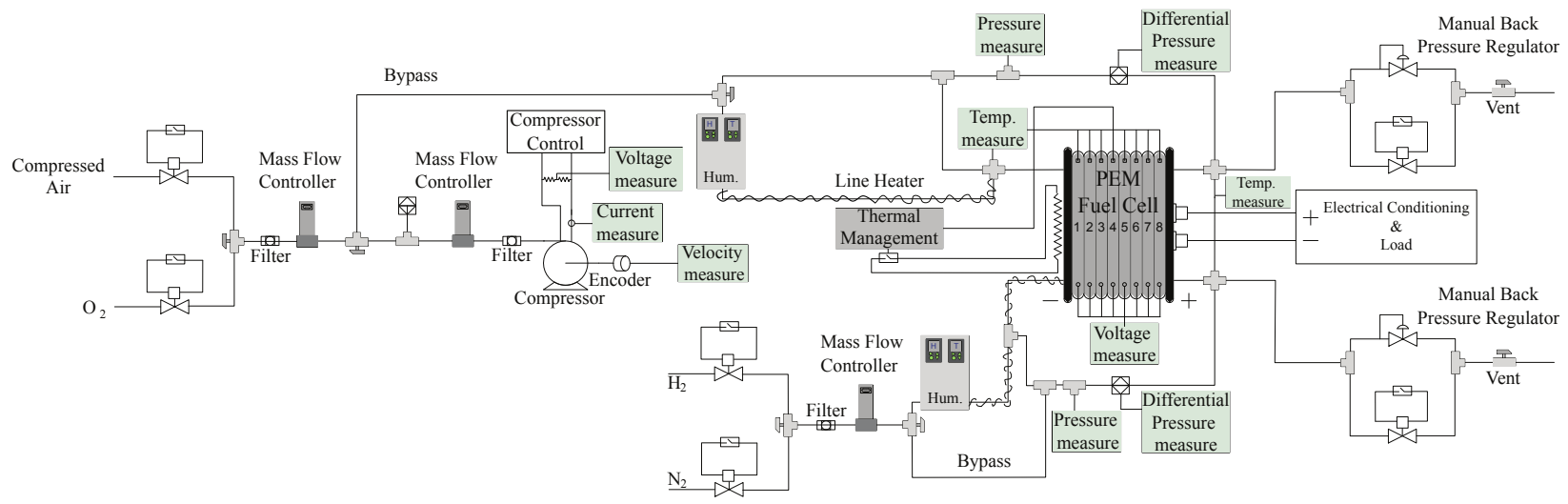

Fig. 1. Schematic diagram of the PEMFC-based generation system.

power. The inner regulation loop is robustly solved using the super twisting controller (a continuous higher-order slidingmode controller). See [2]. In this previous work, the extremum seeking solution is presented as an open problem.

It should also be stressed that the anode subsystem is not considered in the proposed control loop (as it does not affect the inner loop dynamics of the cathode line).

It is also important to stress that, due to the fact that $W_{O_{2}, c a}$ is an internal unavailable variable of the system, it is not practical to include it in the control algorithm. This problem was circumvented by inferring information of $W_{\mathrm{O}_{2}, c a}$ from an accessible variable of the system, such as the air mass flow delivered by the compressor $W_{c p}$. See (1).

Then, the inner control objective can be expressed as $S(x)=0$, where $S(x)=W_{c p}-W_{c p, r e f}$ is the sliding variable that must be steered to zero,

$$
\begin{aligned}
W_{c p} & =B_{00}+B_{10}\left(x_{2} \frac{T_{\text {hum }, c a} R_{a}}{V_{\text {hum }}}+K_{\text {hum }}\right)+ \\
& +B_{20}\left(x_{2} \frac{T_{\text {hum }, c a} R_{a}}{V_{\text {hum }}}+K_{\text {hum }}\right)^{2}+B_{01} x_{1}+ \\
& +B_{11}\left(x_{2} \frac{T_{\text {hum }, c a} R_{a}}{V_{\text {hum }}}+K_{\text {hum }}\right) x_{1}+B_{02} x_{1}^{2}
\end{aligned}
$$

is the compressor air mass flow, $x_{1}=\omega_{c p}$ the compressor speed, $x_{2}=m_{a, \text { hum }}$ the humidifier mass of air and $W_{c p, r e f}$ is the compressor flow reference. The compressor parameters $B_{00}, B_{01}, B_{10}, B_{11}, B_{02}$ and $B_{20}$ can be obtained from [17], $T_{\text {hum }, c a}$ is the humidifier temperature, $V_{\text {hum }}$ is the humidifier volume, $R_{a}$ the air gas constant and $K_{\text {hum }}=P_{\text {sat }}\left(T_{\text {hum }, c a}\right) R H_{\text {hum }}-P_{\text {sat }}\left(T_{a m b}\right) R H_{\text {amb }}$ with $P_{\text {sat }}\left(T_{\text {hum }, c a}\right)$ the vapour saturation pressure at $T_{\text {hum }, c a}$, $R H_{\text {hum }}$ the relative humidity of the gas at the humidifier output, $P_{s a t}\left(T_{a m b}\right)$ the vapour saturation pressure at ambient temperature and $R H_{\text {hum }}$ the relative humidity of ambient air. The robust stabilization problem of setting $S(x)=0$ was solved through a Super-Twisting algorithm as proposed in [2].

In this context, to optimize the system hydrogen consumption for different power loads $\left(P_{n e t}\right)$, the computation of the optimal $W_{c p, r e f}$ in uncertain conditions should be carried out by an extremum seeking algorithm.

The objective of the case study is to optimize the hydrogen consumption of the fuel cell system in every operating con- dition, minimizing the stack current demand under different loads. Note that the consumed hydrogen in the reaction $\left(W_{H_{2}, r e}\right)$ is directly related to the stack current $\left(I_{s t}\right)$,

$$
W_{H_{2}, r e}=G_{H_{2}} \frac{n I_{s t}}{2 F},
$$

where $G_{H_{2}}$ stands for the molar mass of hydrogen, $n$ is the total number of cells of the stack and $F$ is Faraday's constant. In turn, the stack current can be determined from the power relation $I_{s t}=\frac{P_{s t}}{V_{s t}}$, where $P_{s t}$ is the total power delivered by fuel cell stack and $V_{s t}$ is the fuel cell stack voltage. Note that $I_{s t}=\left(P_{n e t}+I_{c p} V_{c p}\right) / V_{s t}$. On the other hand, $V_{s t}, I_{c p}$ and $V_{c p}$ depend indirectly on $W_{c p}$, through the equilibrium equations of the system internal state variables [18]. Therefore, the optimization procedure can be stated as the problem of minimizing the real-valued objective function

$$
I_{s t}=H\left(W_{c p}\right),
$$

where for notational simplicity we have omitted the dependence that $H$ has on $P_{n e t}$ and the system parameters. Further details of the model, assumptions and operating conditions can be found in [12].

The steady state map of the system (the static relationship between $W_{c p}$ and $I_{s t}$ at different values of the net power $P_{n e t}$ ) is depicted in Fig. 2.

Notice that low air mass flow implies low stack voltage and, hence, higher stack current in order to deliver the required $P_{n e t}$. At the same time, a higher air mass flow would require a higher compressor current, which would also increase $I_{s t}$. Thus, if continuity holds, there must be a minimizing value of air mass between the two extrema of air mass flow. Indeed, the input-output maps depicted in Fig. 2 show that such minima exist. What is more, the maps can be reasonably approximated by convex functions. These results were obtained at fixed operating conditions of inlet gases humidities, temperature and hydrogen stoichiometry. Nevertheless, similar results showing the existence of global minima were obtained in different working conditions.

Note that the real nature of the relation between $W_{c p}$ and $I_{s t}$ is dynamical, and the true state-space is infinite-dimensional. The approximation to a static map is valid as long as the extremum seeker (to be defined below) is slow enough for 


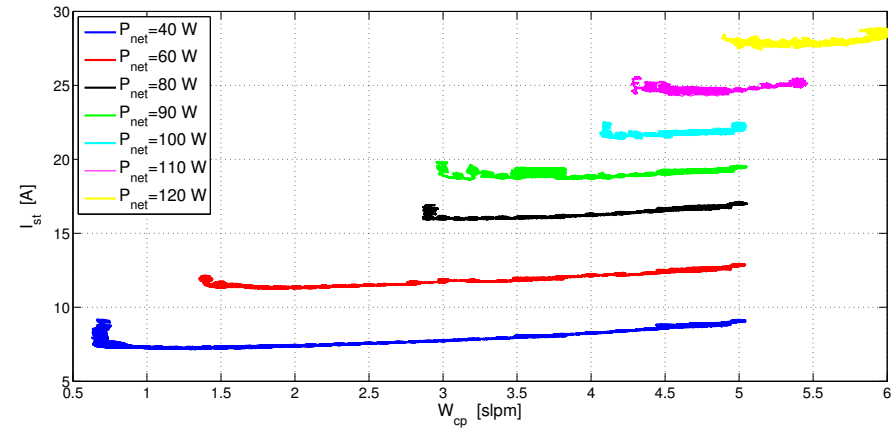

Fig. 2. Steady-state response of the system under different load conditions. Oxygen mass flow vs. stack current. The stack current is proportional to the hydrogen consumption. Experimental data.

the fuel cell dynamics to be considered as instantaneous. This principle has been verified experimentally in [19].

\section{AdAPtive EXTREMUM SEEKING}

In this section we construct a nonsmooth seeker for obtaining the compressor mass flow $\left(W_{c p}\right)$ that achieves the minimal stack current (hence minimal hydrogen consumption) in the presence of plant parametric uncertainty and without explicit knowledge of the power demanded by the load. Please note that the extremum seeking algorithm will compute $W_{c p, r e f}$, while a faster inner control loop based on [2] robustly ensures that $W_{c p}=W_{c p, r e f}$. In terms of time constants of the overall fuel cell system system, the extremum seeking algorithm will have slower dynamics, so the separation principle can be applied and $W_{c p}=W_{c p, r e f}$ can be considered as a static relationship. In the following, also a convergence to the optimal compressor mass flow is proved using an explicit nonsmooth Lyapunov function.

\section{A. Problem statement}

Consider the process (3) and let us denote by $H^{\prime}$ and $H^{\prime \prime}$, respectively, the first and second derivatives of $H$ with respect to $W_{c p}$. The objective is to devise an extremum seeker that finds the optimal mass flow

$$
W_{c p}^{*}:=\underset{W_{c p} \in \mathbb{R}}{\operatorname{argmin}} H\left(W_{c p}\right) .
$$

More precisely, we seek a dynamic system of the form

$$
\begin{aligned}
\dot{\xi} & =g\left(\xi, I_{s t}, \dot{I}_{s t}\right) \\
W_{c p} & =k\left(\xi, I_{s t}, \dot{I}_{s t}\right)
\end{aligned}
$$

such that $W_{c p} \rightarrow W_{c p}^{*}$ as $t \rightarrow \infty$. Here, $\xi \in \mathbb{R}^{m}$ is the controller internal state. The derivative $\dot{I}_{s t}$ is required for estimating $H^{\prime}$ and can be obtained, e.g., with the use of a super-twisting differentiator [20].

\section{B. Rationale}

If $H$ was perfectly known and convex, then a simple nonsmooth steepest descent algorithm

$$
\begin{aligned}
\dot{\xi}_{1} & =-\alpha_{1} \operatorname{sign} H^{\prime}\left(\xi_{1}\right) \\
W_{c p} & =\xi_{1}
\end{aligned}
$$

with $\alpha_{1}>0$ would suffice to accomplish the objective. The problem, however, is more challenging because $H$ is highly uncertain (recall that it depends on the fuel cell parameters and the power load). We only impose the following assumption.

Assumption 1: The function $H: \mathbb{R} \rightarrow \mathbb{R}$ is twice continuously differentiable and satisfies

$$
0<\delta_{1} \leq H^{\prime \prime}\left(W_{c p}\right)<\delta_{2}
$$

for some (possibly unknown) constant $\delta_{1}$, for some known constant $\delta_{2}$ and for all $W_{c p}$ in the domain of interest.

This implies strict convexity, a common assumption in the optimization literature. Notice from Figure 2 that this is a fairly reasonable assumption.

To further simplify the notation, let us define $w:=$ $H^{\prime}\left(W_{c p}\right)$. It is clear that, given the convexity of $H$, our problem reduces to that of finding $W_{c p}^{*}$ such that $\left.w\right|_{W_{c p}=W_{c p}^{*}}=0$. Since $w$ is unknown, we propose to estimate it by borrowing ideas from adaptive control.

Differentiation of (3) gives $\dot{I}_{s t}=w \dot{W}_{c p}$. Using $\dot{I}_{s t}$ and $\dot{W}_{c p}$ we can construct the error $\varepsilon=\dot{I}_{s t}-\xi_{2} \dot{W}_{c p}$, where $\xi_{2}$ is an estimate of $w$. The product $\varepsilon \dot{W}_{c p}=\left(w-\xi_{2}\right) \dot{W}_{c p}^{2}$ contains valuable information about the sign of the estimation error $w-\xi_{2}$ and suggests the estimation law

$$
\dot{\xi}_{2}=\alpha_{2} \operatorname{sign}\left(\varepsilon \dot{W}_{c p}\right)
$$

with $\alpha_{2}>0$.

\section{The nonsmooth extremum seeker}

The extremum seeker is obtained by substituting the equation $\varepsilon \dot{W}_{c p}=\dot{I}_{s t} \dot{W}_{c p}-\xi_{2} \dot{W}_{c p}^{2}$ in (7) and replacing $H^{\prime}\left(\xi_{1}\right)$ by its estimate $\xi_{2}$ in (6a). This leads to the proposed nonsmooth extremum seeker

$$
\begin{aligned}
\dot{\xi}_{1} & =-\alpha_{1} \operatorname{sign} \xi_{2} \\
\dot{\xi}_{2} & =\alpha_{2} \operatorname{sign}\left(\dot{I}_{s t}\left(-\alpha_{1} \operatorname{sign} \xi_{2}\right)-\xi_{2}\left(\alpha_{1} \operatorname{sign} \xi_{2}\right)^{2}\right) \\
W_{c p} & =\xi_{1},
\end{aligned}
$$

the solutions of which are taken in Filippov's sense [21] (see the Appendix for the definition and a short collection of results on nonsmooth Lyapunov analysis).

The following theorem shows that $W_{c p}$ converges to the optimum value and gives explicit conditions for the controller gains $\alpha_{1}$ and $\alpha_{2}$.

Theorem 2: Consider a process (3) satisfying Assumption 1 and set

$$
\alpha_{2}>\frac{\alpha_{1} \delta_{2}}{1-a}>0
$$

with $a$ any constant in the open interval $(0,1)$. Then, $\left(W_{c p}^{*}, 0\right)$ is a globally asymptotically stable equilibrium of (8). Moreover,

$$
L(\xi)=\left|H^{\prime}\left(\xi_{1}\right)-\xi_{2}\right|+a\left|\xi_{2}\right|
$$

is a Lyapunov function.

Proof: Let us begin with the coordinate transformation $\zeta=\zeta(\xi)$, where

$$
\begin{aligned}
& \zeta_{1}=H^{\prime}\left(\xi_{1}\right)-\xi_{2} \\
& \zeta_{2}=\xi_{2} .
\end{aligned}
$$


Note that, because of the assumption on the strict convexity of $H, H^{\prime}$ is a diffeomorphism and the coordinate transformation is well defined. Let us write system (8) in the new coordinates as $\dot{\zeta}=z(\zeta)$. It is straightforward to verify that the system reduces to the autonomous system

$$
\begin{aligned}
& \dot{\zeta}_{1}=-\alpha_{1} H^{\prime \prime}\left(\xi_{1}\right) \operatorname{sign} \zeta_{2}-\alpha_{2} \operatorname{sign} \zeta_{1} \\
& \dot{\zeta}_{2}=\alpha_{2} \operatorname{sign} \zeta_{1},
\end{aligned}
$$

where we have left the term $H^{\prime \prime}$ as a function of $\xi_{1}=$ $\left(H^{\prime}\right)^{-1}\left(\zeta_{1}+\zeta_{2}\right)$ in order to maintain the simplicity of the equations. Still under the same coordinate transformation, the Lyapunov function candidate (10) becomes

$$
V(\zeta):=\left.L(\xi)\right|_{\xi=\xi(\zeta)}=\left|\zeta_{1}\right|+a\left|\zeta_{2}\right|,
$$

with $\xi(\zeta)$ the inverse of $\zeta(\xi)$. The function is positive definite, proper and convex (and Lipschitz continuous and regular in consequence [22]).

To prove asymptotic stability we will show that $\dot{\bar{V}}$, the set derivative of $V$ (see [23] or the Appendix), is zero when $\zeta=0$ and is contained in the open negative semi-axis otherwise. Globality follows from the properness of $W$. We will analyze four different cases. Let us first consider the case where $\zeta_{1} \neq 0$ and $\zeta_{2} \neq 0$. The set-valued derivative is the singleton

$$
\begin{aligned}
\dot{\bar{V}}(\zeta)=\left\{-\alpha_{1} H^{\prime \prime}\left(\xi_{1}\right) \operatorname{sign} \zeta_{2} \cdot\right. & \operatorname{sign} \zeta_{1}-\alpha_{2} \\
& \left.+a \alpha_{2} \operatorname{sign} \zeta_{1} \cdot \operatorname{sign} \zeta_{2}\right\}
\end{aligned}
$$

It follows from Assumption 1 and (9) that

$$
\max \dot{\bar{V}}(\zeta) \leq-\alpha_{2}(1-a)+\alpha_{1} \delta_{2}<0 .
$$

Now, when $\zeta_{1}=0$ and $\zeta_{2} \neq 0$, the set-valued vector field and the generalized gradient are

$K[z](\zeta)=\left(\begin{array}{c}-\alpha_{1} H^{\prime \prime}\left(\xi_{1}\right) \operatorname{sign} \zeta_{2} \\ 0\end{array}\right)+\overline{c o}\left\{\left(\begin{array}{c}-\alpha_{2} \\ \alpha_{2}\end{array}\right),\left(\begin{array}{c}\alpha_{2} \\ -\alpha_{2}\end{array}\right)\right\}$

and

$$
\partial V(\zeta)=\overline{\operatorname{co}}\left\{\left(\begin{array}{c}
-1 \\
a \operatorname{sign} \zeta_{2}
\end{array}\right),\left(\begin{array}{c}
1 \\
a \operatorname{sign} \zeta_{2}
\end{array}\right)\right\} .
$$

The only vector $\eta \in K[z](\zeta)$ such that $v^{\top} \eta$ remains constant for all $v \in \partial V(\zeta)$ (cf. (14)) is $\eta^{\top}=\left(\begin{array}{ll}0 & -\alpha_{1} H^{\prime \prime}\left(\xi_{1}\right) \operatorname{sign} \zeta_{2}\end{array}\right)$, so $\dot{V}(\zeta) \stackrel{\text { a.e. }}{=} v^{\top} \eta=-a \cdot \alpha_{1} H^{\prime \prime}\left(\xi_{1}\right)<0$.

Let $\zeta_{1} \neq 0$ and $\zeta_{2}=0$. We have

$$
\begin{aligned}
K[z](\zeta)=\left(\begin{array}{c}
-\alpha_{2} \operatorname{sign} \zeta_{1} \\
\alpha_{2} \operatorname{sign} \zeta_{1}
\end{array}\right) & \\
& +\overline{\mathrm{co}}\left\{\left(\begin{array}{c}
-\alpha_{1} H^{\prime \prime}\left(\xi_{1}\right) \\
0
\end{array}\right),\left(\begin{array}{c}
\alpha_{1} H^{\prime \prime}\left(\xi_{1}\right) \\
0
\end{array}\right)\right\}
\end{aligned}
$$

and

$$
\partial V(\zeta)=\overline{\operatorname{co}}\left\{\left(\begin{array}{c}
\operatorname{sign} \zeta_{1} \\
-a
\end{array}\right),\left(\begin{array}{c}
\operatorname{sign} \zeta_{1} \\
a
\end{array}\right)\right\} .
$$

For $v^{\top} \eta$ to be constant with respect to all $v \in \partial V(\zeta), \eta$ has to be of the form $\eta^{\top}=\left(\begin{array}{ll}\eta_{1} & 0\end{array}\right)$, but such $\eta$ does not belong to $K[z](\zeta)$. We conclude that $\dot{\bar{W}}=\emptyset$. In other words, the derivative of $V$ does not exist in the case $\zeta_{1} \neq 0$ and $\zeta_{2}=0$. It follows from Lemma 7 that the trajectories necessarily cross the switching line $\zeta_{2}=0$ (no sliding modes occur in this case).

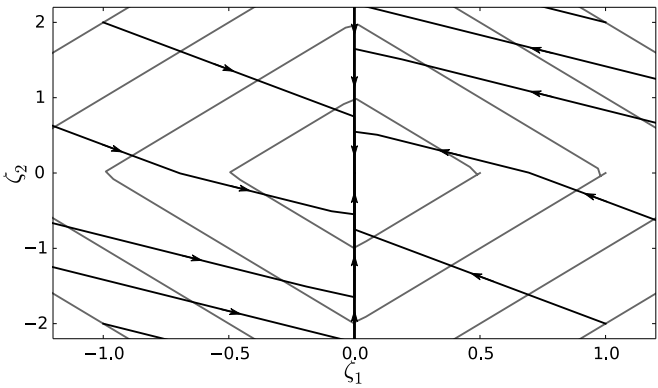

Fig. 3. Phase plane of (12) for a quadratic function $H$. Level curves of $W(\zeta)$ are shown in gray. Notice that the system exhibits a stable sliding mode on the switching line $\zeta_{1}=0$ but trajectories cross the switching line $\zeta_{2}=0$. All trajectories converge to the origin.

Finally, let us consider the case $\zeta=0$. The generalized gradient is

$$
\partial V(\zeta)=\overline{\mathrm{co}}\left\{\left(\begin{array}{l}
-1 \\
-a
\end{array}\right),\left(\begin{array}{c}
1 \\
-a
\end{array}\right),\left(\begin{array}{c}
-1 \\
a
\end{array}\right),\left(\begin{array}{l}
1 \\
a
\end{array}\right)\right\},
$$

from which is clear that $\eta=0$. Since $0 \in K[z](\zeta)$, we have $\dot{\bar{V}}(\zeta)=\{0\}$.

Summarizing: $\max \dot{\bar{V}}(\zeta) \leq 0$, which proves the stability of $\zeta=0$ (Theorem 8). Asymptotic stability follows from the fact that $\{0\}=\left\{\zeta \in \mathbb{R}^{m} \mid 0 \in \dot{\bar{V}}(\zeta)\right\}$ (Theorem 3 in [22]). To illustrate the asymptotic behavior of $\zeta$, we have included in Figure 3 the phase plane of (12) for a quadratic function $H$ along with a few level curves of $V(\zeta)$.

Finally, it follows from (11) that the globally asymptotically stable equilibrium $\zeta=0$ corresponds, in the original coordinates, to $\xi_{2}=0$ and $H^{\prime}\left(\xi_{1}\right)=0$. In view of the convexity of $H$, we have that $H^{\prime}\left(\xi_{1}\right)=0$ implies that $\xi_{1}=W_{c p}^{*}$.

\section{RESULTS}

In the following, the effectiveness of the proposed novel nonsmooth adaptive sliding-mode extremum seeking algorithm presented in section III is illustrated by setting up a realistic scenario for a PEM fuel cell stack, where the seeker plays a decisive role in the optimization of the system efficiency.

\section{A. Experimental Scenario}

In order to evaluate the performance of the proposed extremum seeking scheme, realistic scenarios were considered for covering the entire system operating range, taking into account standard working conditions specified by the fuel cell stack manufacturer. In this context, the following general operation mode was imposed to determine the system inputoutput map:

- A mass flow control device ensures a constant hydrogen stoichiometry supply.

- An auxiliary control system efficiently regulates gas temperatures at five points of the plant: cathode and anode humidifiers $\left(70^{\circ} \mathrm{C}\right)$, cathode and anode line heaters $\left(75^{\circ} \mathrm{C}\right)$ and stack $\left(80^{\circ} \mathrm{C}\right)$.

- A humidity control loop regulates the water injection of the humidifiers to a relative level close to $100 \%$. 
- The water entering the cathode and anode is only in the vapor phase.

The next step was to impose different load conditions to the system and then vary the stack net power $\left(P_{n e t}\right)$ accordingly. The resulting stack current $\left(I_{s t}\right)$ was then recorded for a wide range of compressor flows $\left(W_{c p}\right)$. The raw static data from the test is depicted in Fig. 2. The objective was to build a representative input-output model from the system, based on the real static map of the fuel cell test bench. This represents a fundamental step to intensively test the seeker algorithm, because not all the working conditions (specially extreme) are allowed in practice. It is important to highlight that in a fuel cell test bench there are strict security layers that do not enable the system to work in extreme operating conditions, like close to oxygen starvation.

\section{B. Extremum Seeking Results}

The first test was conducted considering nominal ideal conditions on the plant. A stack net power of $40 \mathrm{~W}$ was imposed and the seeker was tuned following (9). Fig. 4 shows a trajectory of the closed-loop system (seeker plus fuel cell system), projected onto the $W_{c p}-I_{s t}$ plane. A time series of $I_{s t}$ is also presented. The figure confirms that, given an arbitrary initial condition to the fuel cell input $\left(W_{c p}\right)$, the extremum seeking algorithm follows the expected behaviour of asymptotically minimizing the output $I_{s t}$. Note that Fig. 4 presents an extreme test, because the initial condition on $W_{c p}$ is far from the expected final value. As it will become clear in the following, the figure provides an illustrative image of the system behaviour in general, in the sense that the seeker always keeps the same robust behaviour in all working conditions.
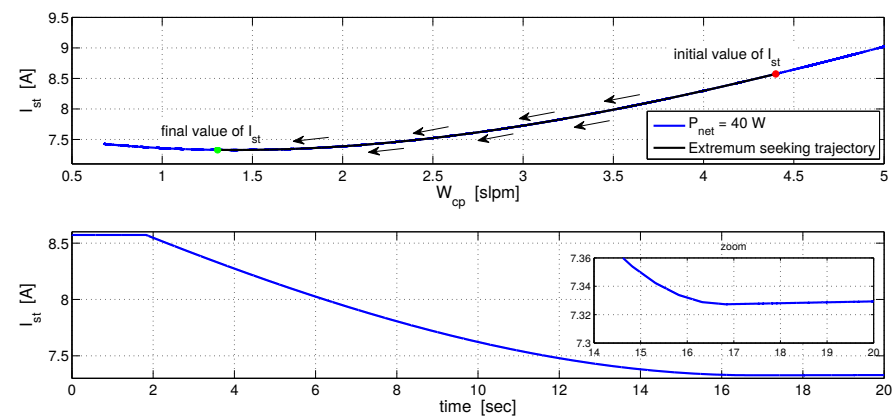

Fig. 4. Closed-loop system evolving on the plane $P_{n e t}=40$. Oxygen mass flow vs. stack current (above). Time vs. Stack current (below). Simulation over experimental data.

Then, a more general set of tests was performed. In this case, the stack net power was varied from $40 \mathrm{~W}$ to $120 \mathrm{~W}$, the entire operation range allowed by the stack manufacturer. Fig. 5 shows the data obtained from the tests. The input-output map is presented in colored lines and the trajectories driven by the seeker are over-imposed in black.

Fig. 6 presents a more illustrative picture of the system behaviour when steered by the seeker. In this case, the static $W_{c p}-I_{s t}$ map, when determined for different power loads, is represented through a two-dimensional surface in the

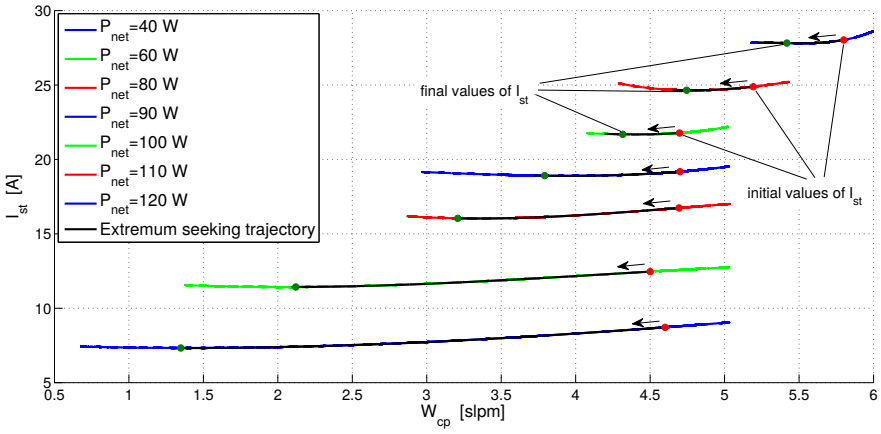

Fig. 5. Closed-loop system trajectories, projected onto the $W_{c p}-I_{s t}$ plane. The input-output map is presented in colored lines and the trajectories driven by the seeker are over-imposed in black. Simulation over experimental data.

$P_{n e t}-W_{c p}-I_{s t}$ space. Some trajectories are also shown in black. The projections of the trajectories onto the $W_{c p}-I_{s t}$ plane are included for better illustration purposes. Note that, although different initial conditions and working scenarios, the extremum seeking control always steers $I_{s t}$ to its minimum.

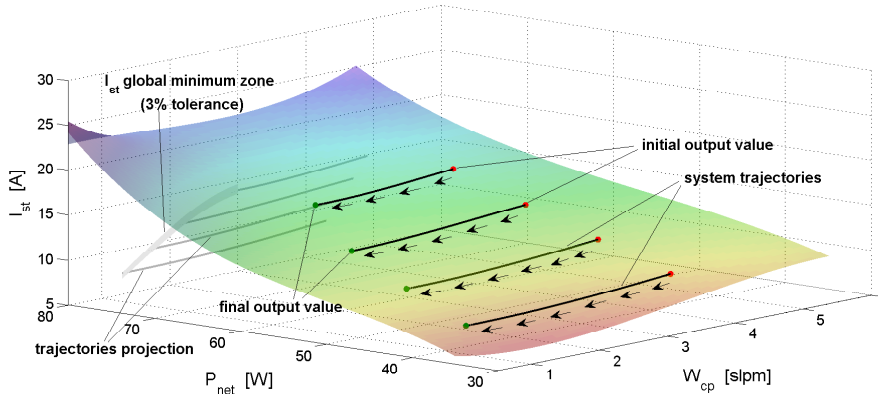

Fig. 6. The static $W_{c p}-I_{s t}$ map, when measured for different power loads, is represented a two-dimensional surface in the $P_{n e t}-W_{c p}-I_{s t}$ space. The map was constructed by interpolating experimental data. Simulation of closed-loop trajectories are shown for several values of $P_{n e t}$. Their projection onto the $W_{c p}-I_{s t}$ plane shows that they converge to the optimal values.

The method was compared against the sliding-mode algorithm with dither [18] and the classical Blackman scheme analyzed in [6]. Three performance indicators are identified in [9]: Domain of convergence (the set of initial conditions for which the closed-loop system converges to the optimal value), rate of convergence and accuracy (quantified by the radius of the ball centered at the optimal value and to which the system trajectories converge). Fig. 7 shows that, in average, all algorithms converge to the optimal value, but the new algorithm converges considerably faster. Moreover, it follows from the Lyapunov analysis that convergence is in finitetime (continuous algorithms such as Blackman's can only ensure asymptotic convergence). As expected from its ditherless nature, the new algorithm exhibits no ripple (the accuracy is perfect).

All the algorithms were tuned by hand, searching for a good compromise between speed of convergence and ripple amplitude. Blackman's algorithm and the sliding-mode algorithm with dither can be made to converge faster, but at the expense of higher ripple (the intricate trade-off between convergence, domain of attraction and accuracy is thoroughly explained in [9]). For further comparison against Blackman's scheme, 

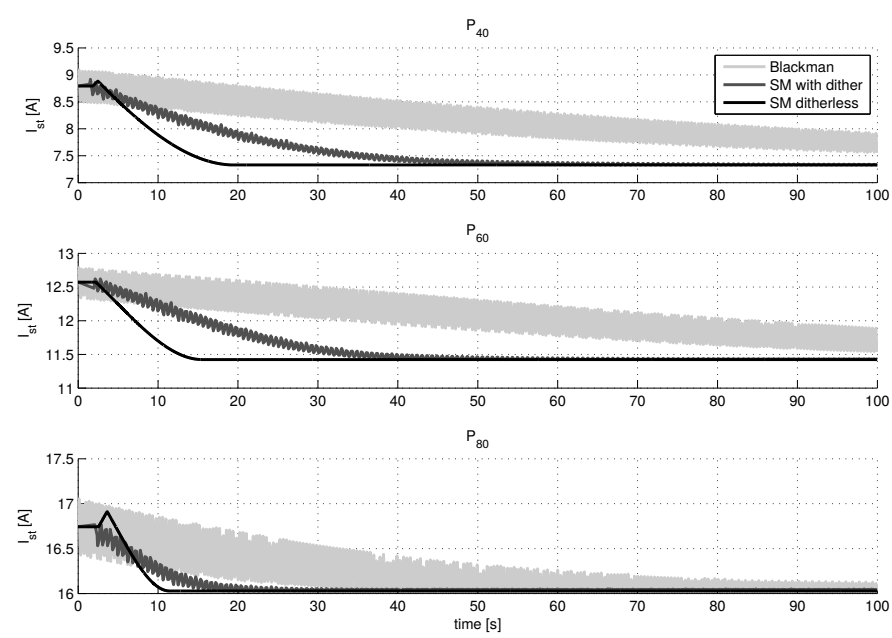

Fig. 7. Comparison between Blackman's algorithm [6], a dither-based slidingmode algorithm [18] and the proposed dither-less extremum seeker. Time vs. Stack current. The performance of the algorithms is shown for different power loads: 40, 60 and $80 \mathrm{~W}$ (from top to bottom). Simulation over experimental data.

the reader is referred to [11].

To conclude the set of tests, a more comprehensive and realistic test was performed. In this case, output noise and actuator dynamics were included. The measurement noise reflects the experimental data presented in Fig. 2, while the added dynamics are linear and of the first order, recalling the Hammerstein and Wiener modeling procedure. This dynamics time constant was set to $\tau=0.2 \mathrm{~s}$, which corresponds to the worst case scenario for the compressor behaviour [12]. The gains were chosen small enough so that overshoots do not drive the system outside its safe operating range. The time-responses might seem slow compared with the fuelcell time-constant. This is due to the time-scale separation principle under which extremum-seekers operate (that is, the time constant for the extremum seeker has to be orders of magnitude away from that of the plant). This is the principle which allows the system to function properly under extreme uncertainty conditions. It can be verified that the seeker still drives the fuel cell to its optimal operating condition.

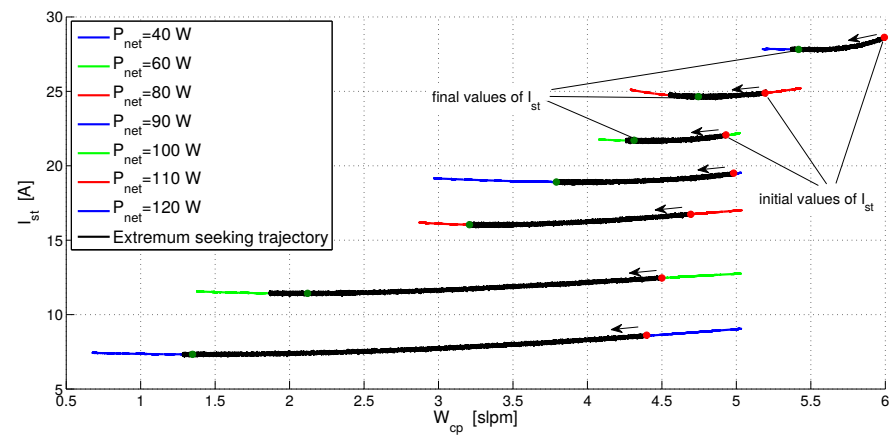

Fig. 8. Closed-loop system trajectories, projected onto the $W_{c p}-I_{s t}$ plane. The input-output map is presented in colored lines and the trajectories driven by the seeker are over-imposed in black. The plant model is composed of a static map (built from experimental data) cascaded with a first-order linear system having a time constant of $2 \mathrm{~s}$. Additive noise is included to emulate the real curves depicted in Fig. 2.

\section{CONCLUSiOns}

A novel nonsmooth adaptive sliding-mode extremum algorithm has been proposed to minimize the hydrogen consumption in PEM fuel cell based systems. The nonsmooth nature of the algorithm avoids the use of dither signals. This allows for convergence to, theoretically, the optimal value. This stands in sharp contrast with extremum seekers for which the dither signal impedes the output of interest to reach the optimal value exactly, and for which in consequence only convergence to a neighborhood can be expected.

Under reasonable convexity assumptions, a nonsmooth Lyapunov function has been used to prove the proper functioning of the extremum seeker. Lyapunov analysis on this function also gives conditions for appropriately tuning the seeker gains. Moreover, the Lyapunov function can in principle be used to study the behavior of the fuel cell when interconnected with other subsystems.

The proposed algorithm was tested using an input-output map (i.e., compressor mass flow vs. stack current) obtained from real experimental data. It can be concluded that, even in the presence of noise and of high uncertainty on the system parameters and power demand (note that $P_{\text {load }}$ is determined a posteriori), the algorithm manages to steer the system to its optimal operating condition. For the lowest power load $(40 \mathrm{~W})$ the current can be reduced from $9 \mathrm{~A}$ to $7.5 \mathrm{~A}$, which amounts to a reduction of more than $15 \%$ (cf. Fig. 8). According to (2), this saving corresponds to a hydrogen flow reduction of $125 \mu \mathrm{g} / \mathrm{s}$. The algorithm was compared against another sliding-mode algorithm which uses dither signals and against the classical algorithm attributed to Blackman. Its dither-less nature makes the algorithm easier to tune (there are less parameters). It converges faster than the other two and, more importantly, without fast oscillations.

\section{APPENDIX A \\ NONSMOOTH LYAPUNOV ANALYSIS}

Definition 3 ([23]): A vector function $\zeta(\cdot)$ is called a solution of $\dot{\zeta}=z(\zeta)$ on $I=\left[t_{0}, t_{1}\right]$ in the sense of Filippov, if $\zeta(\cdot)$ is absolutely continuous and for almost all $t \in I$

$$
\dot{\zeta} \in K[z](\zeta)
$$

where $K[z](\zeta):=\bigcap_{\delta>0} \bigcap_{\mu(N)=0} \overline{c 0}\{z(B(\zeta, \delta)-N)\}$, $B(\zeta, \delta)$ is the ball of center $\zeta$ and radius $\delta$, $\overline{\text { co }}$ denotes convex closure and $\mu$ the usual Lesbegue measure in $\mathbb{R}^{m}$.

Definition 4 ([24]): A function $f: \mathbb{R}^{m} \rightarrow \mathbb{R}^{n}$ is said to be regular at $\zeta \in \mathbb{R}^{m}$ if for all $v \in \mathbb{R}^{m}$, the usual onesided directional derivative $\lim _{t \rightarrow 0^{+}}(f(\zeta+t v)-f(\zeta)) / t$ exists and is equal to the generalized directional derivative $\lim _{y \rightarrow \zeta} \sup _{t \rightarrow 0+}(f(y+t v)-f(y)) / t$.

Definition 5 ([23]): For a function $V: \mathbb{R}^{m} \rightarrow \mathbb{R}$ that is locally Lipschitz, the generalized gradient of $V$ at $\zeta$ is $\partial V(\zeta)=\overline{\mathrm{co}}\left\{\lim \nabla V\left(\zeta_{i}\right) \mid \zeta_{i} \rightarrow \zeta, \zeta_{i} \notin \Omega_{V}\right\}$, where $\Omega_{V}$ is the set of measure zero where the gradient of $V$ is not defined.

Definition 6 ([22]): A Lyapunov function for (13) is a positive definite, continuous function $V: \mathbb{R}^{m} \rightarrow \mathbb{R}$ such that, for each solution $\zeta(\cdot)$ of (13) on $I$ and all $t_{1}, t_{2} \in I, t_{1} \leq t_{2}$ implies that $V\left(\zeta\left(t_{2}\right)\right) \leq V\left(\zeta\left(t_{1}\right)\right)$. 
Lemma 7 ([22]): Let $\zeta(\cdot)$ be a solution of the differential inclusion (13) and let $V: \mathbb{R}^{m} \rightarrow \mathbb{R}$ be a locally Lipschitz continuous regular function. Then, $(\mathrm{d} / \mathrm{d} t) V(\zeta(t))$ exists almost everywhere (a.e.) and $(\mathrm{d} / \mathrm{d} t) V(\zeta(t)) \stackrel{\text { a.e. }}{\epsilon} \dot{\bar{V}}(\zeta)$, where

$$
\begin{aligned}
& \dot{\bar{V}}(\zeta):=\{d \in \mathbb{R} \mid \exists \eta \in K[z](\zeta) \text { such that } \\
& \left.\qquad v^{\top} \eta=d \text { for all } v \in \partial V(\zeta)\right\} .
\end{aligned}
$$

is the set-valued derivative of $V(\zeta)$.

Theorem 8 ([22]): If $V: \mathbb{R}^{m} \rightarrow \mathbb{R}$ is a positive definite, locally Lipschitz continuous and regular function such that, for all $\zeta \in \mathbb{R}^{m}$, $\max \dot{\bar{V}}(\zeta) \leq 0$, then (13) is stable at $\zeta=0$.

To simplify the exposition, it has been agreed in the pre-

\begin{tabular}{|c|c|c|c|}
\hline \multicolumn{3}{|c|}{ APPENDIX B } & \\
\hline & trical variables & & nperatures \\
\hline $\begin{array}{l}V_{c p} \\
I_{c p} \\
V_{s t} \\
I_{s t} \\
P_{n e t} \\
P_{s t}\end{array}$ & $\begin{array}{l}\text { Compressor voltage } \\
\text { Compressor current } \\
\text { Stack voltage } \\
\text { Stack current (output) } \\
\text { Load power (disturb.) } \\
\text { Stack power }\end{array}$ & $\begin{array}{l}T_{h u m, c a} \\
T_{h u m, a n} \\
T_{l h, a n} \\
T_{l h, c a} \\
T_{\text {st }}\end{array}$ & $\begin{array}{l}\text { Cathode humidifier } \\
\text { Anode humidifier } \\
\text { Anode line heater } \\
\text { Cathode line heater } \\
\text { Stack }\end{array}$ \\
\hline & Mass flows & & scellaneous \\
\hline $\begin{array}{l}W_{c p} \\
W_{\mathrm{O}_{2}, \text { ca }} \\
W_{\mathrm{H}_{2}} \\
W_{\mathrm{H}_{2}, \text { re }}\end{array}$ & $\begin{array}{l}\text { Air (input) } \\
\text { Oxygen } \\
\text { Hydrogen } \\
\text { Consumed hydrogen }\end{array}$ & $\begin{array}{l}P_{\text {sat }} \\
R H_{\text {hum }} \\
\omega_{\text {amb }}\end{array}$ & $\begin{array}{l}\text { Vap. sat. } \\
\text { RH at hum. output } \\
\text { Ambient air } \\
\text { humidity ratio }\end{array}$ \\
\hline \multicolumn{4}{|c|}{ Internal states } \\
\hline$x_{1}, x_{2}$ & Compressor & $\xi_{1}, \xi_{2}$ & Extremum seeker \\
\hline
\end{tabular}
ceding theorem that $\max \dot{\bar{V}}(\zeta)=-\infty$ whenever $\dot{\bar{V}}(\zeta)=\emptyset$.

\section{ACKNOWLEDGEMENTS}

All the experimental tests were performed at the PEM Fuel Cells Laboratory of the Institut de Robòtica i Informàtica Industrial (CSIC-UPC), Barcelona (Spain) and only possible due to its advanced equipment and proficient technical staff.

\section{REFERENCES}

[1] C. Ziogou, S. Papadopoulou, M. C. Georgiadis, and S. Voutetakis, "Online nonlinear model predictive control of a PEM fuel cellsystem," $J$. Process Control, vol. 23, no. 4, pp. 483 - 492, 2013.

[2] C. Kunusch, P. Puleston, M. Mayosky, and L. Fridman, "Experimental results applying second order sliding mode control to a PEM fuel cell based system," Control Eng. Pract., vol. 21, no. 5, pp. 719 - 726, 2013.

[3] J. Pukrushpan, A. Stefanopoulou, and H. Peng, Control of Fuel Cell Power Systems. Springer, 2004.

[4] F. Bianchi, C. Kunusch, C. Ocampo-Martinez, and R.S. Sánchez-Peña, "A gain-scheduled LPV control for oxygen stoichiometry regulation in PEM fuel cell systems," IEEE Trans. Control Syst. Technol., 2014 (In press).

[5] S. Kelouwani, K. Adegnon, K. Agbossou, and Y. Dube, "Online system identification and adaptive control for PEM fuel cell maximum efficiency tracking," IEEE Trans. Energy Convers., vol. 27, no. 3, pp. 580-592, Sept 2012.

[6] M. Krstić and H.-H. Wang, "Stability of extremum seeking feedback for general nonlinear dynamic systems," Automatica, vol. 36, pp. $595-$ $601,2000$.

[7] Y. Tan, W. H. Moase, C. Manzie, D. Nešić, and I. Mareels, "Extremum seeking from 1922 to 2010," in Proc. Chinese Control Conference, Beijing, China, Jul. 2010, pp. $14-26$.

[8] M. Krstić, "Performance improvement and limitations in extremum seeking control," Systems and Control Lett., vol. 39, pp. 313 - 326, 2000.

[9] D. Nešić, "Extremum seeking control: Convergence analysis," European Journal of Control, vol. 15, pp. 331 - 347, 2009.
[10] I. Matraji, F. Ahmed, S. Laghrouche, and M. Wack, "Extremum seeking control for net power output maximization of a PEM fuel cell using second order sliding mode," in Proc. of Variable Structure Systems Workshop, 2012.

[11] Y. A. Chang and S. J. Moura, "Air flow control in fuel cell systems: An extremum seeking approach," in Proc. American Control Conference, St. Louis, MO, Jun. 2009, pp. $1052-1059$.

[12] C. Kunusch, P. Puleston, M. Mayosky, and A. Husar, "Control oriented modelling and experimental validation of a PEMFC generation system," IEEE Trans. Energy Convers., vol. 6, no. 3, pp. 851-861, 2011.

[13] C. Restrepo, T. Konjedic, C. Guarnizo, O. Avino-Salvado, J. Calvente, A. Romero, and R. Giral, "Simplified mathematical model for calculating the oxygen excess ratio of a pem fuel cell system in real-time applications," Industrial Electronics, IEEE Transactions on, vol. 61, no. 6, pp. 2816-2825, June 2014.

[14] A. Arce, A. del Real, C. Bordons, and D. Ramirez, "Real-time implementation of a constrained mpc for efficient airflow control in a pem fuel cell," Industrial Electronics, IEEE Transactions on, vol. 57, no. 6, pp. 1892-1905, June 2010.

[15] C. Ramos-Paja, R. Giral, L. Martinez-Salamero, J. Romano, A. Romero, and G. Spagnuolo, "A pem fuel-cell model featuring oxygen-excess-ratio estimation and power-electronics interaction," Industrial Electronics, IEEE Transactions on, vol. 57, no. 6, pp. 1914-1924, June 2010.

[16] F. Marignetti, M. Minutillo, A. Perna, and E. Jannelli, "Assessment of fuel cell performance under different air stoichiometries and fuel composition," Industrial Electronics, IEEE Transactions on, vol. 58, no. 6, pp. 2420-2426, June 2011.

[17] C. Kunusch, P. F. Puleston, and M. Mayoski, Sliding-Mode Control of PEM Fuel Cells. Springer-Verlag, 2012.

[18] C. Kunusch and F. Castaños, "Extremum seeking algorithms for minimal hydrogen consumption in PEM fuel cells," in Proc. American Control Conference, Washington, DC, USA, Jun. 2013, pp. 1146 - 1151.

[19] - "On the implementation of an adaptive extremum seeking algorithm for hydrogen minimization in PEM fuel cell based systems," in Proc. European Control Conference, Zürich, Switzerland, Jul. 2013, pp. $2501-2506$.

[20] A. Levant, "Robust exact differenciation via slding mode technique," Automatica, vol. 34, pp. 379-384, Mar. 1998.

[21] A. F. Filippov, Differential Equations with Discontinuous Righthand Sides. Dordrecht, The Netherlands: Kluwer Academic Publishers, 1988.

[22] A. Bacciotti and F. Ceragioli, "Stability and stabilization of discontinuous systems and nonsmooth lyapunov functions," ESAIM: COCV, vol. 4, pp. $361-376,1999$.

[23] D. Shevitz and B. Paden, "Lyapunov stability theory of nonsmooth systems," IEEE Trans. Autom. Control, vol. 39, pp. 1910 - 1914, 1994

[24] F. H. Clarke, Optimization and Nonsmooth Analisis. New York: Society for Industrial and Applied Mathematics, 1990.

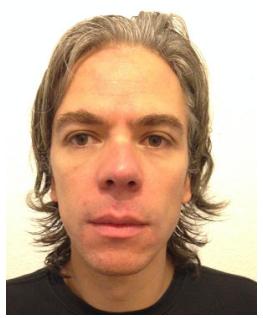

Fernando Castaños was born in Mexico City in 1976. He received the B.Eng. in Electric and Electronic Engineering from Universidad Nacional Autnoma de México (UNAM). He received the M.Eng. in Control Engineering from UNAM and the Ph.D degree from Université Paris-Sud XI. He was a postdoctoral fellow at McGill's Center for intelligent machines for two years. He joined the Automatic Control Department, Cinvestav, Mexico, in 2011. His research interests are passivity-based control, nonlinear control, port-Hamiltonian systems, robust control and variable structure systems.

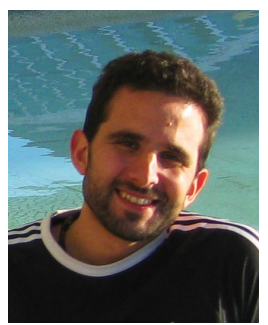

Cristian Kunusch (S'03-M'10) received the B.S., M.Sc., and $\mathrm{Ph} . \mathrm{D}$. degrees in electronic engineering from the National University of La Plata, Buenos Aires, Argentina, in 2003, 2006, and 2009, respectively. In 2010, he was appointed as a Postdoctoral Fellow of the Spanish National Research Council, Institut de Robòtica i Informàtica Industrial, Barcelona, Spain. Between 2011 and 2013 he run a Marie Curie project funded by the European Commission. In 2014, he joined the Electric Drives Advanced Development team of Brose Fahrzeugteile, Würzburg, Germany, as a Senior Researcher. His main research interests include variable structure systems and their applications to the control and observation of fuel-cell-based systems and electric automotive drives. 